\title{
Mapping Three-Dimensional Surface Deformation by Combining Multiple-Aperture Interferometry and Conventional Interferometry: Application to the June 2007 Eruption of Kilauea Volcano, Hawaii
}

\author{
H. S. Jung, Z. Lu, J. S. Won, M. P. Poland, and A. Miklius
}

\begin{abstract}
Surface deformation caused by an intrusion and small eruption during June 17-19, 2007, along the East Rift Zone of Kilauea Volcano, Hawaii, was three-dimensionally reconstructed from radar interferograms acquired by the Advanced Land Observing Satellite (ALOS) phased-array type L-band synthetic aperture radar (SAR) (PALSAR) instrument. To retrieve the 3-D surface deformation, a method that combines multipleaperture interferometry (MAI) and conventional interferometric SAR (InSAR) techniques was applied to one ascending and one descending ALOS PALSAR interferometric pair. The maximum displacements as a result of the intrusion and eruption are about $0.8,2$, and $0.7 \mathrm{~m}$ in the east, north, and up components, respectively. The radar-measured 3-D surface deformation agrees with GPS data from 24 sites on the volcano, and the root-mean-square errors in the east, north, and up components of the displacement are $1.6,3.6$, and $2.1 \mathrm{~cm}$, respectively. Since a horizontal deformation of more than $1 \mathrm{~m}$ was dominantly in the north-northwest-south-southeast direction, a significant improvement of the north-south component measurement was achieved by the inclusion of MAI measurements that can reach a standard deviation of $3.6 \mathrm{~cm}$. A 3-D deformation reconstruction through the combination of conventional InSAR and MAI will allow for better modeling, and hence, a more comprehensive understanding, of the source geometry associated with volcanic, seismic, and other processes that are manifested by surface deformation.
\end{abstract}

Index Terms-Interferometric synthetic aperture radar (SAR) (InSAR), multiple-aperture interferometry (MAI), SAR, 3-D surface deformation measurement.

\section{INTRODUCTION}

$\mathbf{T}$ HE INTERFEROMETRIC synthetic aperture radar (InSAR) technique has demonstrated success in map-

Manuscript received July 22, 2009; revised November 25, 2009, January 30, 2010, and March 22, 2010; accepted May 24, 2010. Date of publication July 8, 2010; date of current version December 27, 2010. This work was supported in part by the National Space Lab Program through the Korea Science and Engineering Foundation funded by the Ministry of Education, Science and Technology (M105DA000004-08D0100-0011A), by the NASA Earth Surface and Interior Program (2005-0021), by the USGS Volcano Hazards Program, and by the USGS Land Remote Sensing Program.

H. S. Jung is with the Department of Geoinfomatics, University of Seoul, Seoul 130-743, Korea.

Z. Lu is with the U.S. Geological Survey, EROS Center and Cascades Volcano Observatory, Vancouver, WA 98683 USA.

J. S. Won is with the Department of Earth System Sciences, Yonsei University, Seoul 120-749, Korea.

M. P. Poland and A. Miklius are with the U.S. Geological Survey, Hawaiian Volcano Observatory, Hawaii National Park, HI 96718-0051 USA.

Color versions of one or more of the figures in this paper are available online at http://ieeexplore.ieee.org.

Digital Object Identifier 10.1109/LGRS.2010.2051793 ping surface deformations (e.g., [1] and [2]) but is limited to measurement of displacements along the radar line-of-sight (LOS) direction. Because SAR satellites have near-polar orbits (i.e., approximately north-south ground tracks and east-west look directions), it is impossible to determine 3-D surface deformation from LOS InSAR data alone, even when multiple independent interferograms with different viewing angles are used jointly [3]. When deformation magnitudes are of several tens of centimeters, it is possible to reconstruct the 3-D deformation field from InSAR using two interferograms generated from ascending and descending pairs and one or two azimuth pixel-offset tracking fields created by cross correlation of SAR images [4]. This method has difficulty in determining the north component of surface deformation due to the low sensitivity of the SAR pixel-offset tracking method (relative to LOS displacement resolution).

Multiple-aperture interferometry (MAI) technique [5], [6] represents a remarkable improvement in measuring alongtrack deformation than the pixel-offset tracking method. While traditional InSAR is most sensitive to vertical and east-west horizontal surface displacements, the MAI method improves the precision of along-track ( north-south) deformation. In this letter, we constructed 3-D deformation by integrating the improved MAI technique [6] and conventional InSAR using one (or more) descending and one (or more) ascending interferogram pairs. We demonstrated the power of this 3-D construction technique by applying the method to the June 17-19, 2007 (Father's Day), intrusion and eruption along the East Rift Zone (ERZ) of Kilauea Volcano [7]. The derived 3-D deformation results capture the dominantly north-northwest-southsoutheast horizontal deformation field along the ERZ and agree well with GPS data spanning the event.

\section{METHOD}

\section{A. Brief Description of MAI Technique}

MAI utilizes subaperture processing techniques to generate one forward-looking and one backward-looking single-lookcomplex (SLC) image from one raw SAR scene [5]. Two raw SAR scenes are then used to form one forward-looking and one backward-looking interferograms using the corresponding SLC images. The phase difference between the forward-looking and the backward-looking interferograms is a MAI image, which 
reveals ground surface deformation in the azimuth direction (approximately north-south). Jung et al. [6] improved MAI generation by correcting MAI interferograms for the phase contributions from the flat Earth and topographic effects and then also proposed an improved MAI processing procedure to enhance coherence as well as to improve computational efficiency. The improved MAI processing [6] is accomplished by the following steps: 1) generation of forward- and backward-looking SLC pairs from raw SAR data by azimuth common-band filtering; 2) creation of forward- and backward-looking differential interferograms using a digital elevation model (DEM) and considering their corresponding perpendicular baselines; and 3) construction of the MAI interferogram by a complexconjugate multiplication of the forward-looking differential interferogram with the backward-looking one.

The MAI interferometric phase $\phi_{\mathrm{MAI}}$ is defined by

$$
\phi_{\mathrm{MAI}}=-\frac{4 \pi}{l} n x
$$

where $x$ is the along-track surface deformation, $l$ is the effective antenna length, and $n$ is a normalized squint that is a fraction of the full aperture width [5]. Although MAI measurement is independent of radar wavelength, it requires that the interferometric pair is coherent. Generally, longer wavelength (e.g., L-band) SAR images maintain higher coherence and consequently, relatively more accurate MAI measurements than shorter wavelength (e.g., C-band and X-band) ones. For Advanced Land Observing Satellites (ALOSs), $l=8.9 \mathrm{~m}$ and one fringe yields $8.9 \mathrm{~m}$ if $n=0.5$ is used.

For a MAI interferogram, the flat-Earth phase contribution is defined by

$$
\frac{\partial \phi_{\mathrm{MAI}}}{\partial \rho} \approx \frac{4 \pi}{\lambda} \cdot \frac{\Delta B_{\perp} \cdot \cos \theta}{\rho \cdot \sin \theta}
$$

where $\lambda$ and $\theta$ are wavelength and look angle, respectively, $\rho$ is slant-range distance, and $\Delta B_{\perp}$ is the difference between the perpendicular baselines for the forward-looking and backwardlooking interferograms [6]. Given nominal system parameters of ALOS phased-array type L-band SAR (PALSAR), the flatEarth phase difference from the near to far range of a SAR image is about $20^{\circ}$ when $\Delta B_{\perp}$ is $0.1 \mathrm{~m}$. Suppose that the MAI is processed using $n=0.5$ and a resulting fringe $\left(360^{\circ}\right)$ is $8.9 \mathrm{~m}$ [as with the previous one from (1)]. In this case, the flat-Earth phase difference corresponds to $50 \mathrm{~cm}$ of apparent along-track deformation. The topographic phase contribution is

$$
\phi_{\mathrm{MAI}}-\phi_{\mathrm{MAI}, f} \approx \frac{4 \pi}{\lambda \rho} \cdot \frac{\Delta B_{\perp}}{\cdot \sin \theta} \cdot h
$$

where $\phi_{\mathrm{MAI}, f}$ is the flat-Earth phase and $h$ is the topographic height. Given the parameters of ALOS PALSAR, a topographic difference of $2000 \mathrm{~m}$ contributes $1.1^{\circ}$ to the MAI phase (or $2.72 \mathrm{~cm}$ in along-track deformation) when $\Delta B_{\perp}$ is $0.1 \mathrm{~m}$.

\section{B. Determination of 3-D Deformations}

Following the notation given by [3], let $d=\left(d_{x}, d_{y}, d_{z}\right)^{\mathbf{T}}$ be the 3-D deformation vector in a local (east, north, up) reference

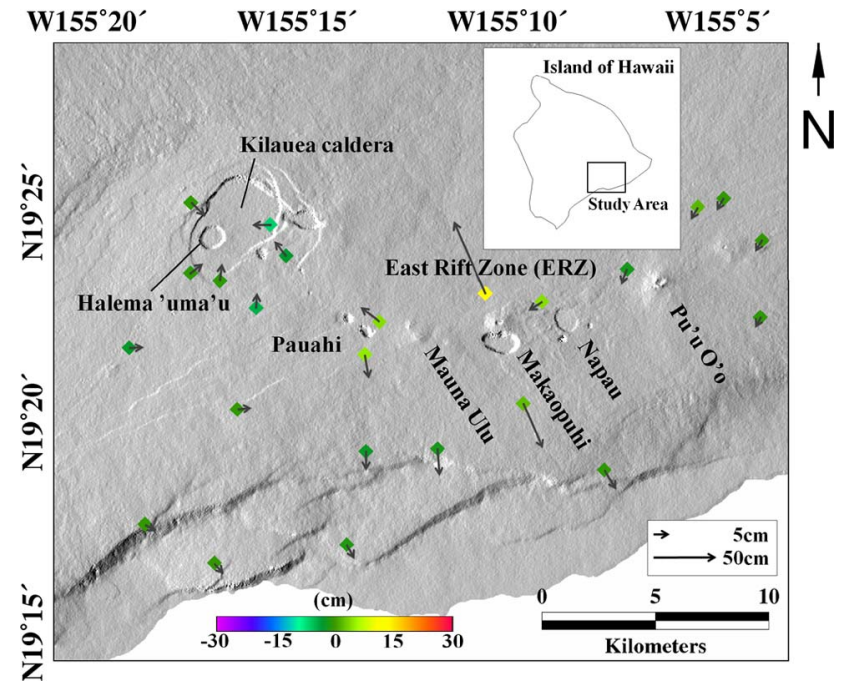

Fig. 1. Shaded relief map for Kilauea Volcano. Campaign and continuous GPS stations are labeled as solid diamonds. The arrows and colors represent the horizontal and the vertical components of surface deformation measured by GPS, respectively.

frame, and $u=\left(u_{x}, u_{y}, u_{z}\right)^{\mathbf{T}}$ be the unit LOS deformation or along-track deformation vector expressed in the same local reference frame. If $u$ is the LOS deformation vector, $u=$ $(-\sin \theta \cdot \cos \varphi, \sin \theta \cdot \sin \varphi, \cos \theta)^{\mathbf{T}}$, where $\theta$ is the radar incidence angle from the vertical and $\varphi$ is the satellite-track angle from north of the LOS vector, respectively. Calculations of $\theta$ and $\varphi$ are performed on a pixel-by-pixel basis. If $u$ is the alongtrack deformation vector, $u=(-\sin \varphi,-\cos \varphi, 0)^{\mathbf{T}}$. The deformation $r$ measured from an InSAR or MAI is given by

$$
r=-u^{\mathbf{T}} d
$$

Suppose we produce a total of $n$ InSAR and MAI interferograms (observations); then, we obtain $R=\left(r_{1}, r_{2}, \ldots, r_{n}\right)^{\mathbf{T}}$. The weighted least square solution $(\hat{d})$ for $d$ is defined as follows:

$$
\hat{d}=-\left(U^{\mathbf{T}} \boldsymbol{\Sigma}^{-1} U\right)^{-1} \cdot\left(U^{\mathbf{T}} \boldsymbol{\Sigma}^{-1} R\right)
$$

where $\Sigma$ is the covariance matrix for errors in the observed deformation observations, and $U$ and $R$ are given by $U=\left(u_{1}, u_{2}, u_{3}, \ldots, u_{n}\right)^{\mathbf{T}}$ and $R=\left(r_{1}, r_{2}, r_{3}, \ldots, r_{n}\right)^{\mathbf{T}}$, respectively.

In the case that we assume the covariance matrix as a diagonal matrix, we get $\boldsymbol{\Sigma}=\operatorname{diag}\left(\sigma_{1}^{2}, \sigma_{2}^{2}, \sigma_{3}^{2}, \ldots, \sigma_{n}^{2}\right)$, where $\operatorname{diag}(\cdot)$ denotes a diagonal matrix. To determine the relative weighting factors for the InSAR and MAI measurements, we estimate the corresponding standard deviations in the InSAR and MAI images. This calculation is performed on a pixel-bypixel basis using a $5 \times 5$ moving window.

\section{APPLICATION}

Since January 1983, the eruptive activity at Kilauea Volcano, Hawaii, has been nearly continuous from Pu'u 'Ô'ô and other vents along the volcano's ERZ [8]. On the morning of June 17, 2007 (Father's Day), a new episode of volcanic activity began 
TABLE I

ChARACTERISTICS OF INTERFEROMETRIC PAIRS USED IN THIS STUdY

\begin{tabular}{|c|c|c|c|c|c|c|c|c|c|}
\hline Pairs & $\begin{array}{l}\text { Acquisition } \\
\text { Date }\end{array}$ & $\begin{array}{l}\text { Beam } \\
\text { Mode }\end{array}$ & $\begin{array}{c}\text { Incidence } \\
\text { Angle } \\
\text { (deg.) }\end{array}$ & $\begin{array}{c}f_{D C, f}{ }^{\mathrm{c}} \\
(\mathrm{Hz})\end{array}$ & $\begin{array}{l}f_{D C, c^{c}} \\
(\mathrm{~Hz})\end{array}$ & $\begin{array}{l}f_{D C, b}{ }^{c} \\
(\mathrm{~Hz})\end{array}$ & $\begin{array}{c}\Delta f_{D, S}{ }^{\mathrm{d}} \\
(\mathrm{Hz})\end{array}$ & $\begin{array}{l}B_{\perp}{ }^{\mathrm{e}} \\
(\mathrm{m})\end{array}$ & $\begin{array}{c}\Delta B_{\perp}{ }^{\mathrm{e}} \\
(\mathrm{m})\end{array}$ \\
\hline Ascending & $\begin{array}{c}05 / 05 / 2007^{\mathrm{a}} \\
20 / 06 / 2007\end{array}$ & $\begin{array}{l}\mathrm{FBD}^{\mathrm{b}} \\
\mathrm{FBD}^{\mathrm{b}}\end{array}$ & 38.7 & 506.0 & 101.7 & -302.0 & 802.8 & 326 & 0.1 \\
\hline Descending & $\begin{array}{c}28 / 02 / 2007^{\mathrm{a}} \\
16 / 07 / 2007\end{array}$ & $\begin{array}{l}\text { FBD }^{\mathrm{b}} \\
\text { FBS }^{\mathrm{b}}\end{array}$ & 38.7 & 489.7 & 85.6 & -318.4 & 650.4 & 260 & -0.4 \\
\hline
\end{tabular}
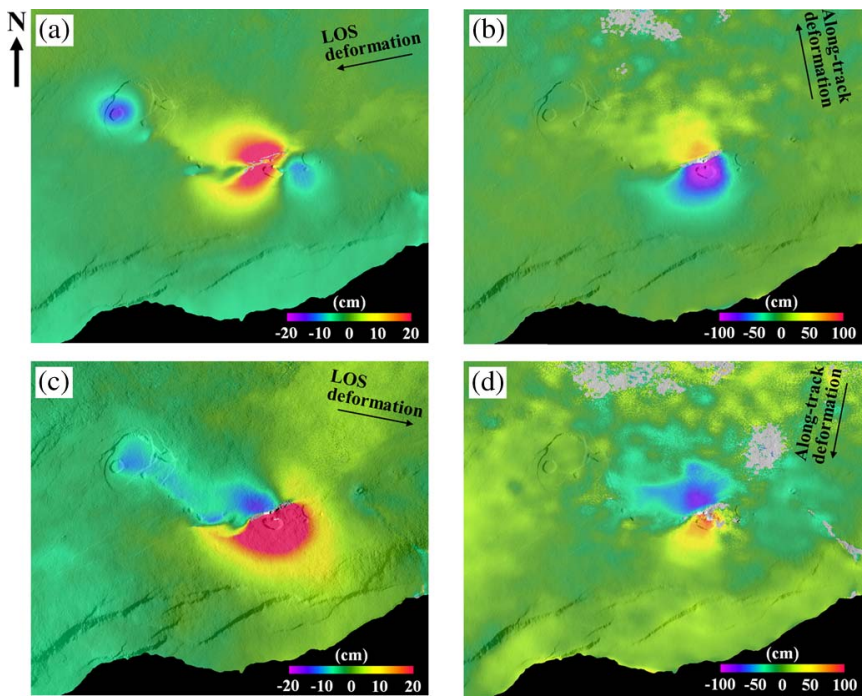

Fig. 2. LOS and along-track surface deformation measured from (a) and (b), InSAR and MAI interferograms of ALOS PALSAR ascending pair acquired on May 5, 2007, and June 20, 2007, and (c) and (d), InSAR and MAI interferograms of ALOS PALSAR descending pair acquired on February 28, 2007 and July 16, 2007.

as rapid deflation at the Kilauea's summit and an increase in earthquake activity. Simultaneously, ground tilt in the vicinity of Mauna Ulu indicated a magmatic intrusion along the ERZ about halfway between the summit and Pu'u 'Ô'ô (Fig. 1). A small eruption occurred just north of Makaopuhi Crater on June 18-19 as a result of the intrusion, and by the late morning of June 19 (local time), the summit began to inflate, signaling an end to the activity [7].

We resolved the 3-D surface deformation associated with the June 2007 intrusion and eruption along the ERZ of Kilauea Volcano by combining the InSAR and MAI techniques. We used the ALOS PALSAR ascending and descending orbit pairs (see Table I). In the MAI interferograms, the flat-Earth phase distortions were $22.7^{\circ}$ and $90.7^{\circ}$ (or 56.1 and $224.3 \mathrm{~cm}$ in the along-track direction) in the ascending and descending pairs, respectively. The topographic phase distortions were $1.1^{\circ}$ and $4.5^{\circ}$ (or 2.8 and $11.1 \mathrm{~cm}$ in the along-track direction) in the ascending and descending pairs, respectively. We corrected these apparent flat-Earth and topographic phase distortions using the method proposed by [6].

Both an InSAR image and an MAI interferogram were obtained from each interferometric pair using a complex multilook operation, with four looks in the range direction and 16 looks in the azimuth direction, resulting in a pixel dimension of about $60 \mathrm{~m}$ by $50 \mathrm{~m}$ in ground-range and azimuth directions, respectively. For MAI processing, the forward- and backwardlooking SLC images were generated using a normalized squint of $n=0.5$ and an effective antenna length of $l=8.9$, and the final MAI interferograms were formed by the complex-conjugate operation of the forward- and backward-looking differential interferograms created using the corresponding perpendicular baselines and a DEM. The processing parameters of the interferometric pairs are summarized in Table I.

Fig. 2 shows LOS and along-track deformation maps measured from InSAR and MAI interferograms for the ascending and descending pairs. Deflation at Kilauea's summit and uplift due to dike intrusion between Mauna Ulu and Makaopuhi Crater are clear in the LOS surface-deformation maps [Fig. 2(a) and (c)], and opening of the upper ERZ is obvious from azimuth surface deformation [Fig. 2(b) and (d)]. The time spans in the ascending and descending pairs are 46 and 138 days, respectively. Despite the difference in time spans, the deformation apparent in the ascending interferogram is similar to that of the descending one because the surface-deformation field is dominated by the June 17-19, 2007 intrusion and eruption [7].

We determined the east $\left(u_{x}\right)$, north $\left(u_{y}\right)$, and up $\left(u_{z}\right)$ components of 3-D deformation vectors from two LOS InSAR measurements and two along-track MAI measurements. We calculated the standard deviations in the InSAR and MAI using a $5 \times 5$ moving window on a pixel-by-pixel basis. The average standard deviations of the ascending InSAR and MAI measurements were 1.0 and $3.6 \mathrm{~cm}$, respectively, and those of the descending InSAR and MAI measurements were 2.3 and $9.7 \mathrm{~cm}$, respectively. We then constructed the covariance matrix in order to determine the relative weighting for the two LOS InSAR measurements and two along-track MAI measurements. This inversion was performed on a pixel-by-pixel basis. Fig. 3 shows the estimated 3-D deformation field for the Kilauea's June 2007 intrusion and eruption. Fig. 4 shows the 3-D deformation along a profile across the ERZ where opening due to dike intrusion was the largest. Within a zone of $500 \mathrm{~m}$ over the center of the intrusion, no deformation can be obtained due to loss of InSAR coherence. Figs. 3 and 4 show a classic picture of surface deformation due to dike opening. The maximum displacements as a result of the intrusion and eruption are about $0.8,2$, and $0.7 \mathrm{~m}$ in the east, north, and up components, respectively.

In order to assess the overall accuracy of the 3-D surfacedeformation field derived from our method of combining InSAR and MAI techniques, we compared the InSAR/ MAI-derived deformation with that from GPS data that span the June 2007 intrusion and eruption. The 3-D deformation images 

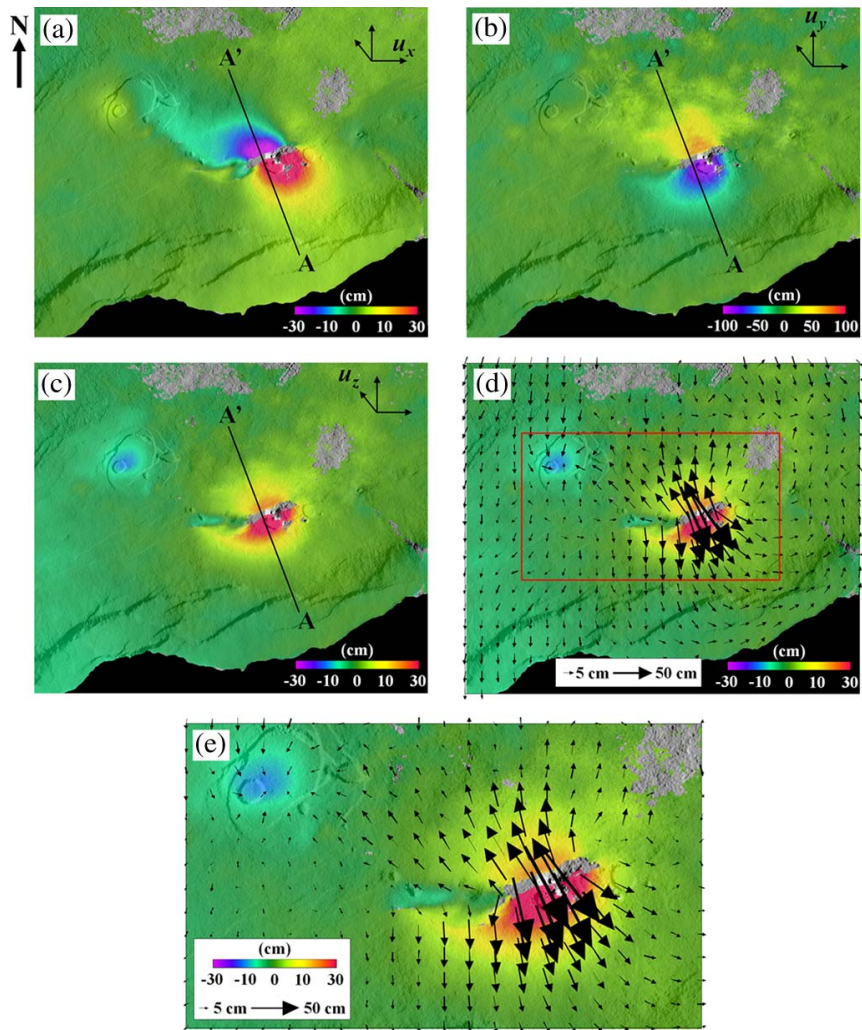

Fig. 3. Three-dimensional surface-deformation field for the Father's Day intrusion and eruption measured from two LOS and two along-track MAI images: (a) East $\left(u_{x}\right)$, (b) north $\left(u_{y}\right)$, and (c) up $\left(u_{z}\right)$ components of deformation field constructed by the integration of MAI and InSAR images. (d) The 3-D deformation field. (e) Detailed blow-up view of the near-field deformation in (d). Arrows and colors represent the horizontal and vertical components, respectively.

along with the SAR backscattering intensity images were precisely georeferenced to a geographic coordinate system through a terrain-correction procedure (Fig. 3). We then extracted the InSAR/MAI-derived 3-D surface-deformation values at the GPS stations using spline interpolation and compared them with the 3-D GPS components. The GPS data come from both campaign and continuous sites. Serendipitously, the campaign sites were occupied only days before June 17, 2007, and were occupied again immediately following the cessation of activity; thus, the GPS campaign data include only deformation that is a result of the June 17-19, 2007 intrusion and eruption. Displacements at continuous GPS sites were similarly constrained. Fig. 5 shows the comparison between InSAR/MAI results and 3-D deformation measurements from 24 continuous and campaign GPS stations (Fig. 1). The root-mean-square errors (rmses) between InSAR/MAI and GPS in the east, north, and up components are 1.6, 3.6, and $2.1 \mathrm{~cm}$, respectively. The agreements between InSAR/MAI and GPS in the east and up components are better than that for the north component because the determination of the east and up components is dominated by the InSAR LOS deformation, which is more precise than the along-track deformation estimate from MAI. The relatively large rmse in the up component (compared with that for the east component) is because the precision of the horizontal component in the GPS data is better than that of the vertical component.

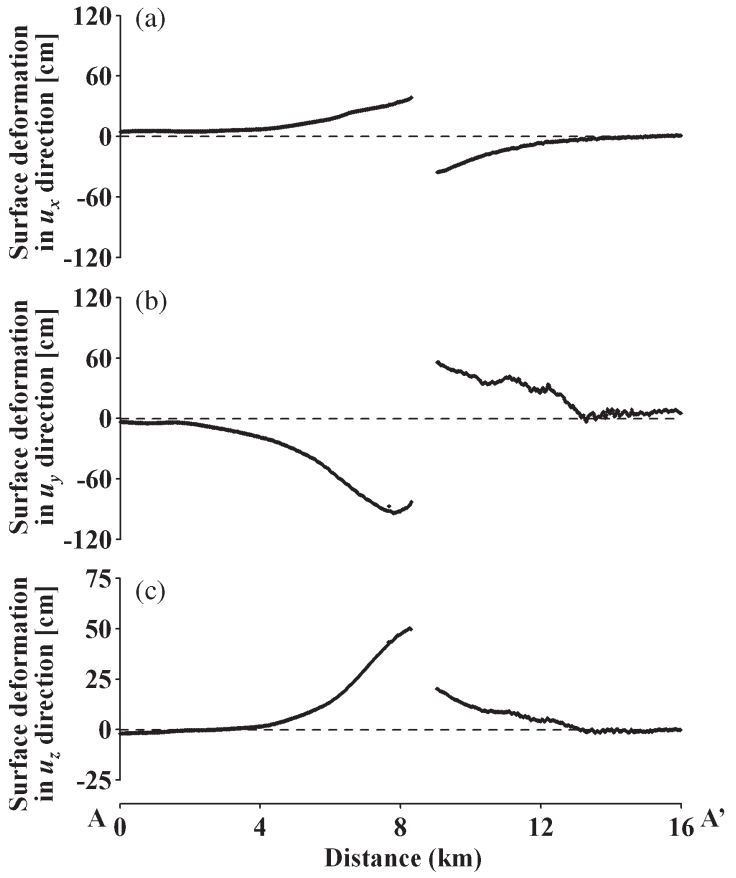

Fig. 4. (a) East $\left(u_{x}\right)$, (b) north $\left(u_{y}\right)$, and (c) up $\left(u_{z}\right)$ components of surface deformation along the profile $\mathrm{A}-\mathrm{A}^{\prime}$ shown in Fig. 3.

The presented method is a significant improvement upon the 3-D displacement estimates from four interferograms with different viewing geometries. Compared with other 3-D deformation-construction techniques [9], our method can derive 3-D deformation images from two pairs of SAR images alone and does not assume that the derived deformation field be smooth. The improvement in our InSAR/MAI result is primarily due to the MAI technique, which can measure the alongtrack deformation more precisely. For example, the standard deviation of along-track deformation of the ascending pair estimated by MAI is $3.57 \mathrm{~cm}$, which is significantly less than the $7.1 \mathrm{~cm}$ achieved by pixel-offset tracking of the same interferogram [10]. Furthermore, MAI does not depend on SAR image resolution, while the azimuth pixel-offset method relies on SAR azimuth pixel resolution. This has significant implication for measuring along-track deformation from future SAR missions. For example, the main operational mode for European Space Agency's Sentinel-1 radar mission [11] has an azimuth pixel resolution of $20 \mathrm{~m}$, which is about five times worse than those for Envisat or ALOS PALSAR. Therefore, the precision of azimuth deformation from the pixel-offset tracking of Sentinel-1 images will be significantly reduced. However, the reduction in SAR azimuth resolution will not significantly affect the azimuth-deformation measurement derived from MAI. Therefore, we expect that the precision of along-track deformation from MAI processing of Sentinel-1 images will be five-ten times better than that from the pixel-offset tracking method.

The 3-D surface-deformation images estimated from the combination of InSAR and MAI techniques will allow for better resolution of the parameters of deformation models for earthquakes, volcanic activity, and other processes, including the geometry of the deformation source. In particular, for the dominantly north-south horizontal deformation, the resolution 
(a)

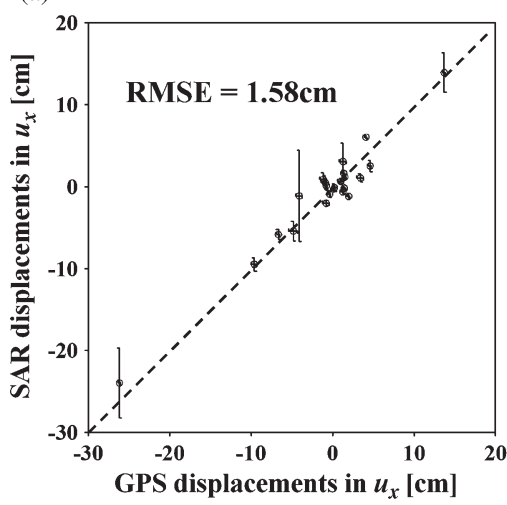

(b)

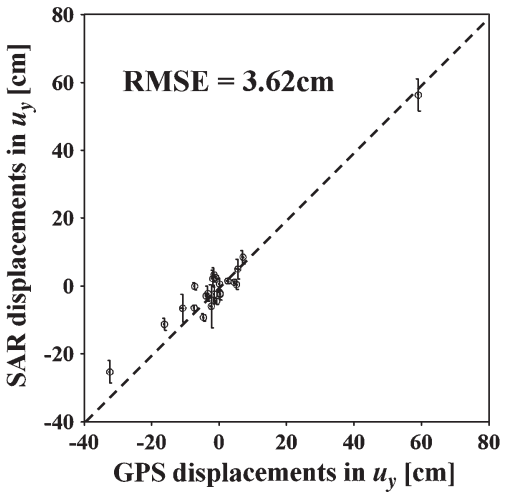

(c)

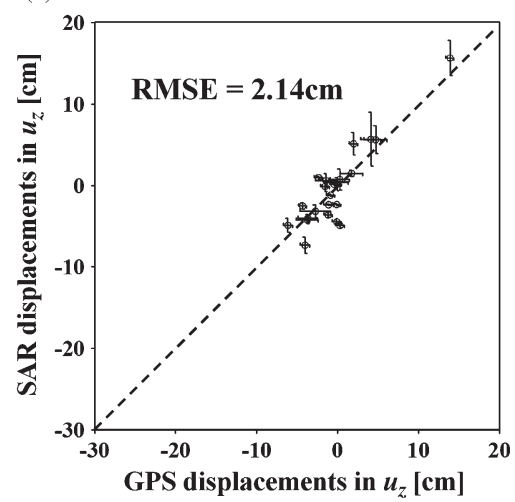

Fig. 5. Comparison of 3-D deformation estimated from ALOS InSAR and MAI techniques with GPS displacements at 24 stations on Kilauea Volcano. (a) East component. (b) North component. (c) Up component. Circles and bars denote uncertainties of InSAR/MAI and GPS measurements at two standard deviations, respectively.

of deformation model parameters would be improved. Source geometry is ambiguous if it is estimated from verticaldeformation data alone [12]; thus, the combination of techniques to recover 3-D displacements with the high spatial resolution of InSAR offers important constraints that can be used to resolve deformation sources at a higher level than has previously been possible.

\section{CONCLUSION}

We have shown that 3-D surface deformation can be recovered with a high degree of accuracy from ascending and descending SAR data using InSAR and MAI techniques. Our estimates of 3-D deformation associated with the June 17-19, 2007, ERZ intrusion and eruption at Kilauea Volcano agree with the GPS data spanning the event, with rmses of 1.6, 3.6, and $2.1 \mathrm{~cm}$ in the east, north, and up components, respectively. The remarkable improvement of accuracy in the north component over other techniques is primarily due to the improved deformation measurement in the north-south direction by MAI technique. A 3-D deformation mapping by combining InSAR and MAI techniques will provide data that can result in improved deformation source models for various applications.

\section{ACKNOWLEDGMENT}

PALSAR data are copyrighted by JAXA/ME 2007 and were provided by the Alaska Satellite Facility. The continuous GPS network on Kilauea Volcano is jointly operated by the USGS Hawaiian Volcano Observatory, Pacific GPS Facility at the University of Hawaii and Stanford University.

\section{REFERENCES}

[1] D. Massonnet and K. L. Feigl, "Radar interferometry and its application to changes in the earth's surface," Rev. Geophys., vol. 36, no. 4, pp. 441-500, Nov. 1998.

[2] Z. Lu, O. Kwoun, and R. Rykhus, "Interferometric synthetic aperture radar (InSAR): Its pasts, present and future," Photogramm. Eng. Remote Sens., vol. 73, no. 3, pp. 217-221, Mar. 2007.

[3] T. J. Wright, B. E. Parsons, and Z. Lu, "Toward mapping surface deformation in three dimensions using InSAR," Geophys. Res. Lett., vol. 31, no. 1, p. L01 607, Jan. 2004, DOI:10.1029/2003GL018827.

[4] Y. Fialko, M. Simons, and D. Agnew, "The complete (3-D) surface displacement field in the epicentral area of the 1999 Mw 7.1 Hector Mine earthquake, California, from space geodetic observations," Geophys. Res. Lett., vol. 28, no. 16, pp. 2063-3066, Aug. 2001.

[5] N. B. D. Bechor and H. A. Zebker, "Measuring two-dimensional movements using a single InSAR pair," Geophys. Res. Lett., vol. 33, no. 16, p. L16311, Aug. 2006, DOI:10.1029/2006GL026883.

[6] H.-S. Jung, J.-S. Won, and S.-W. Kim, "An improvement of the performance of multiple aperture SAR interferometry (MAI)," IEEE Trans. Geosci. Remote Sens., vol. 47, no. 8, pp. 2859-2869, Aug. 2009.

[7] M. P. Poland, A. Miklius, T. Orr, J. Sutton, C. Thornber, and D. Wilson, "New episodes of volcanism at Kỉlauea Volcano, Hawaii," EOS Trans. $A G U$, vol. 89, no. 5, pp. 37-38, Jan. 2008.

[8] C. Heliker and T. N. Mattox, "The first two decades of the Pu-u O'oKupaianaha eruption: Chronology and selected bibliography," in The Pu'u O'o-Kupaianaha Eruption of Kilauea Volcano, Hawaii: The First 20 Years, C. Heliker, D. A. Swanson, and T. J. Takahashi, Eds. Washington, DC: U.S. Geol. Surv., 2003, pp. 1-27.

[9] S. Samsonov, K. Tiampo, J. Rundle, and Z. Li, "Application of DInSARGPS optimization for derivation of fine scale surface motion maps of southern California," IEEE Trans. Geosci. Remote Sens., vol. 45, no. 2, pp. 512-521, Feb. 2007.

[10] D. Sandwell, D. Myer, R. Mellors, M. Shimada, B. Brooks, and J. Foster, "Accuracy and resolution of ALOS interferometry: Vector deformation maps of the Father's Day intrusion at Kilauea," IEEE Trans. Geosci. Remote Sens., vol. 46, no. 11, pp. 3524-3534, Nov. 2008.

[11] P. Snoeij, E. Attema, A. M. Guarnieri, and F. Rocca, "GMES Sentinel-1 FDBAQ performance analysis," in Proc. Radar Conf., 2009, pp. 1-6.

[12] J. H. Dieterich and R. W. Decker, "Finite element modeling of surface deformation associated with volcanism," J. Geophys. Res., vol. 80, no. B29, pp. 4094-4102, 1975. 